\section{How can next-generation diagnostics aid pancreatic adenocarcinoma treatment?}
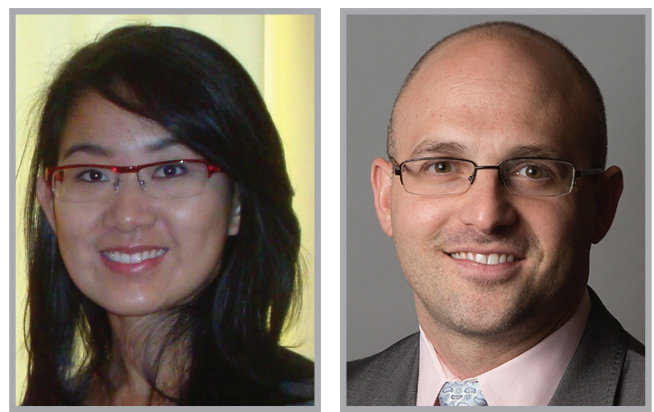

\author{
“Implementing useful \\ prognostic or predictive \\ biomarkers for routine use in \\ pancreatic ductal \\ adenocarcinoma has posed a \\ great challenge.”
}

Shuang Qin Zhang' \& Daniel VT Catenacci*,1

First draft submitted: 1 December 2015; Accepted for publication: 14 December 2015; Published online: 2 February 2016

\section{Pancreatic cancer: prognosis}

Pancreatic ductal adenocarcinoma (PDAC) has the poorest prognosis of all solid tumors, as reflected by a 5 -year survival rate of less than $5 \%$ and a median survival of $8-11$ months. It is characterized by a high rate of local recurrence, distant metastasis and chemotherapy-resistant phenotype. While the majority of patients present with metastatic disease, only approximately $20 \%$ of patients with PDAC present with localized disease and are potentially appropriate for surgical resection with curative intent. After resection, these patients have a high propensity to suffer local and/or distant metastatic recurrence with a 5 -year overall survival rarely exceeding $20 \%$ [1].

\section{Molecular profiling \& targeted} therapies

With such dismal prognosis, advancing our understanding of pancreatic cancer genetics and proteomics could substantially improve therapy efficacy. It is now well accepted that most malignancies progress from genomic alterations that dysregulate critical molecular pathways and confer tumorigenesis [2]. Identification of these alterations and associated pathways can generate clinically relevant diagnostic, prognostic and therapeutic information. Targeting a specific genetic alteration is already commonplace in numerous scenarios $[3,4]$. These are testimony to how acquisition of genetic information can successfully be translated to clinical management. However, classic low-throughput companion diagnostics are challenged by limited tissue and tumor heterogeneity, suggesting a role for a general implementation of next-generation higher-throughput molecular diagnostics, including nextgeneration sequencing (NGS) and mass spectrometry (MS) on tumor samples along with applying these technologies to 'liquid biopsies' $[4,5]$.

\section{Challenges of incorporating companion diagnostics for pancreatic cancer \\ In contrast, implementation of molecu- lar biomarkers for prognostic and/or}

'Department of Medicine, Section of Hematology \& Oncology, University of Chicago, Chicago, IL 60637, USA *Author for correspondence: Tel.: +1 773702 7596; Fax: +1 773702 3163; dcatenac@bsd.uchicago.edu

\section{KEYWORDS}

- next-generation sequencing

- pancreatic cancer
"While the majority of patients present with metastatic disease, only approximately $20 \%$ of patients with pancreatic ductal adenocarcinoma present with

localized disease and are potentially appropriate for surgical resection with curative intent."

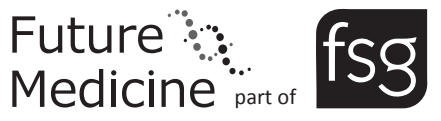




\section{"Various strategies are being implemented to address the numerous hurdles faced with attempting to validating potential prognostic and predictive biomarkers and therapies."}

predictive guidance for pancreatic cancer has been elusive to date with no required assay incorporated into routine standard care. This is despite numerous potential prognostic and therapeutic biomarkers which have been explored. For instance, potential predictive biomarkers for cytotoxic therapy, such as putative predictors for gemcitabine or nab-paclitaxel responsiveness, have ultimately been discouraging [6]. A number of reasons contribute to the lack of prognostic and predictive biomarkers in PDAC, including many of the same heterogeneity and tissue limitation challenges facing all cancers [7].

First, limited tissue is particularly challenging in localized disease within the pancreas and compromises the ability to acquire adequate tissue for testing and validating biomarker utility (for both low- and high-throughput assays). The anatomical location of the pancreas and inherent intratumoral/stromal heterogeneity presents unique barriers to optimal specimen acquisition, testing and data interpretation. At present, in the locally advanced setting, tissue is typically acquired via endoscopic ultrasound (EUS)-guided fine-needle aspiration obtaining very few cells. Additionally, the proportion of viable tumor and nontumor cells in a given sample affects sensitivity of genetic mutation and proteomic profiling. PDAC is characterized by substantial desmoplastic stroma, posing significant technical challenges for next-generation tissue diagnostics [2].

Second, in PDAC, the genetic profile on typical oncogene panels ( $\sim 300$ genes) appears to be particularly homogeneous in general with the majority of tumors being KRAS driven. Oncogenic mutant KRAS is present in up to $90 \%$ of PDAC and is the most frequent and among the earliest genetic aberrations in pancreatic tumorigenesis $[8,9]$. To date, effective targeted therapies for KRAS-driven tumors are lacking in PDAC and other cancers, with ongoing efforts to identify druggable downstream or synergistic effectors of KRAS, or novel siRNA therapeutics that may provide clinical benefit in the future $[10,11]$. Supporting homogeneity, a PDAC study used capillary-based exome sequencing and single nucleotide polymorphism (SNP) microarrays to identify genetic alterations in both cell lines and xenografts derived from primary and metastatic tumors of 24 patients [8]. Results revealed that aside from the wellrecognized and predominant genetic alterations (KRAS activation and inactivation of TP53, CDKN2A and SMAD) [9,12], the prevalence of other aberrations with potential functional consequences was less than $5 \%$. Conversely, in a different study, whole-genome sequencing of PDAC revealed more genetic heterogeneity than previously reported with diverse molecular subtypes described [8,13-14]. Moreover, analyses of metastatic lesions even within the same patient have revealed different mutational profiles, suggesting an ongoing and parallel genomic evolution $[2,15]$. Thus, upon more expansive profiling with nextgeneration diagnostics, it is possible that in the future more molecular subsets within PDAC may be validated to assist in understanding varying prognoses and therapeutic possibilities.

\section{Next-generation companion diagnostics}

\section{for the future}

Difficulties of adequate tissue acquisition and the inherent genomic heterogeneity of disease, particularly with evolution after therapy, have led to a number of potential solutions. Improvement in the technical ability of various next-generation technologies to provide results from smaller tissue samples has shown great promise. For example, NGS on tissue DNA from cells obtained via fineneedle aspiration is now feasible clinically [16]. Moreover, circulating tumor cells (CTCs) and/or circulating tumor DNA (ctDNA) have arisen as noninvasive molecular profiling strategies [17]. Although peripheral pancreatic CTC enumeration may be a predictive and/or prognostic indicator of clinical outcomes [18,19], peripheral CTCs are quite rare in pancreatic cancer, with an estimate of one tumor cell per 1 billion circulating blood cells, and generally not reliably detectable until disease is widely metastatic. Due to biophysical factors, specifically possible hepatic filtration CTCs during transit through the portal circulation, we evaluated the feasibility and safety of sampling portal venous blood by EUS to enumerate portal venous CTCs of pancreatibiliary patients and compare to CTC numbers in the peripheral blood [20]. EUS acquisition of portal vein blood was determined to be feasible and safe, and significantly higher numbers of CTCs were identified in the portal system compared with the peripheral system; genomic and proteomic tumor profiling of the acquired cells was also demonstrated. This novel and far more sensitive way to acquire, enumerate and characterize CTCs from pancreatobiliary, and other gastrointestinal cancers for similar reasons, may be most applicable in the potential ability to better risk stratify patients being considered for 
curative intent surgery, where peripheral blood CTCs would be unrevealing. For example, in the perioperative setting, those patients identified to have no (or low) CTCs in the portal vein versus those that have higher numbers may have different prognoses. It is also possible that particular molecular signatures would convey a better or worse prognosis. With this understanding, treatments may then be personalized accordingly. However, definitive prospective studies need to be performed to confirm these hypotheses with larger numbers of patients in a controlled setting. Finally, serial molecular evaluation using CTCs and/or ctDNA [21] from the peripheral or portal venous systems may provide an opportunity to molecularly profile patients for immuno-oncologic signatures in a minimally invasive manner over time in attempt to address molecular evolution with potential implications on treatment decisions in the future, if validated.

\section{Diagnostic validation \& next-generation clinical trials}

In order to validate these various biomarkers and next-generation diagnostics, prospective next-generation clinical trial designs currently place particular emphasis on patient selection based on genetic profile [4]. Various strategies are being implemented to address the numerous hurdles faced with attempting to validating potential prognostic and predictive biomarkers and therapies. In the National Cancer Institute (NCI) MATCH trial, patients are screened to receive targeted drug combination based on the tumor's genetic profile, independent of tumor histology [22]. In the NCI-MPACT trial, patients receive either genetic mutation-specific therapy or nonpathway-specific therapy [23]. Notably, a recent setback for matching targeted therapies based on putative biomarker information was recently observed in the histology agnostic 'personalized therapy' or 'expansion platform type II' SHIVA trial [24]. However, a number of factors must be considered from this type of trial, and refinements in the diagnostics used, therapies incorporated and treatment strategy imposed will provide for a platform for many future investigations [25]. These future trials, both for histology agnostic and PDAC-specific scenarios, will further test the value of putative biomarkers and their next-generation companion diagnostics for clinical utility.

\section{Summary}

Implementing useful prognostic or predictive biomarkers for routine use in PDAC has posed a great challenge. Application of next-generation diagnostics, including NGS, MS and CTC and ctDNA approaches, has great potential to change our understanding of the biology along with prognostic and therapeutic guidance in the future. Validation of next-generation diagnostics coupled with next-generation clinical trials is underway.

\section{Financial \& competing interests disclosure}

The authors have no relevant affiliations or financial involvement with any organization or entity with a financial interest in or financial conflict with the subject matter or materials discussed in the manuscript. This includes employment, consultancies, honoraria, stock ownership or options, expert testimony, grants or patents received or pending, or royalties.

No writing assistance was utilized in the production of this manuscript.

\section{Open access}

This work is licensed under the Creative Commons Attribution-NonCommercial 4.0 Unported License. To view a copy of this license, visit http://creativecommons.org/ licenses/by-nc-nd/4.0/

\section{References}

1 Siegel R, Naishadham D, Jemal A. Cancer statistics, 2013. CA Cancer J. Clin. 63(1), 11-30 (2013).

2 Vogelstein B, Papadopoulos N, Velculescu VE, Zhou S, Diaz LA Jr, Kinzler KW. Cancer genome landscapes. Science 339(6127), 1546-1558 (2013).

3 Khoury JD, Catenacci DV. Next-generation companion diagnostics: promises, challenges, and solutions. Arch. Pathol. Lab. Med. 139(1), 11-13 (2015).
Catenacci DV. Next-generation clinical trials: novel strategies to address the challenge of tumor molecular heterogeneity. Mol. Oncol. 9(5), 967-996 (2015).

5 Stricker T, Catenacci DV, Seiwert TY. Molecular profiling of cancer - the future of personalized cancer medicine: a primer on cancer biology and the tools necessary to bring molecular testing to the clinic. Semin. Oncol. 38(2), 173-185 (2011).

6 Poplin E, Wasan H, Rolfe L et al. Randomized, multicenter, Phase II study of CO-101 versus gemcitabine in patients with metastatic pancreatic ductal adenocarcinoma: including a prospective evaluation of the role of hENT1 in gemcitabine or CO-101 sensitivity. J. Clin. Oncol. 31(35), 4453-4461 (2013).

7 Hidalgo M, Plaza C, Musteanu M et al. SPARC expression did not predict efficacy of nab-paclitaxel plus gemcitabine or gemcitabine alone for metastatic pancreatic cancer in an exploratory analysis of the Phase III MPACT Trial. Clin. Cancer Res. 21(21), 4811-4818 (2015).

8 Jones S, Zhang X, Parsons DW et al. Core signaling pathways in human pancreatic 
cancers revealed by global genomic analyses. Science 321(5897), 1801-1806 (2008).

9 Zhang SY, Zhang SQ, Nagaraju GP, El-Rayes BF. Biomarkers for personalized medicine in GI cancers. Mol. Aspects Med. 45, 14-27 (2015).

10 Eser S, Schnieke A, Schneider G, Saur D. Oncogenic KRAS signalling in pancreatic cancer. Br. J. Cancer 111(5), 817-822 (2014).

11 Yuan TL, Fellmann C, Lee CS et al. Development of siRNA payloads to target KRAS-mutant cancer. Cancer Discov. 4(10), 1182-1197 (2014).

12 Oshima M, Okano K, Muraki S et al. Immunohistochemically detected expression of 3 major genes (CDKN2A/p16, TP53, and $S M A D 4 / D P C 4)$ strongly predicts survival in patients with resectable pancreatic cancer. Ann. Surg. 258(2), 336-346 (2013).

13 Biankin AV, Waddell N, Kassahn KS et al. Pancreatic cancer genomes reveal aberrations in axon guidance pathway genes. Nature 491(7424), 399-405 (2012).

14 Cowley MJ, Chang DK, Pajic M et al. Understanding pancreatic cancer genomes.
J. Hepatobiliary Pancreat. Sci. 20(6), 549-556 (2013).

15 Ling S, Hu Z, Yang Z et al. Extremely high genetic diversity in a single tumor points to prevalence of non-Darwinian cell evolution. Proc. Natl Acad. Sci. USA 112(47), E6496-E6505 (2015).

16 Young G, Wang K, He J et al. Clinical next-generation sequencing successfully applied to fine-needle aspirations of pulmonary and pancreatic neoplasms. Cancer Cytopathol. 121(12), 688-694 (2013).

17 De Mattos-Arruda L, Cortes J, Santarpia L et al. Circulating tumour cells and cell-free DNA as tools for managing breast cancer. Nat. Rev. Clin. Oncol. 10(7), 377-389 (2013).

18 Cristofanilli M, Budd GT, Ellis MJ et al. Circulating tumor cells, disease progression, and survival in metastatic breast cancer. N. Engl. J. Med. 351(8), 781-791 (2004).

19 Kurihara T, Itoi T, Sofuni A et al. Detection of circulating tumor cells in patients with pancreatic cancer: a preliminary result. J. Hepatobiliary Pancreat. Surg. 15(2), 189-195 (2008).
20 Catenacci DV, Chapman CG, Xu P et al. Acquisition of portal venous circulating tumor cells from patients with pancreaticobiliary cancers by endoscopic ultrasound. Gastroenterology 149(7), 1794-1803 (2015).

21 Zill OA, Greene C, Sebisanovic D et al. Cell-free DNA next-generation sequencing in pancreatobiliary carcinomas. Cancer Discov. 5(10), 1040-1048 (2015).

22 Clinical trials database: NCT02465060. https://clinicaltrials.gov

23 Clinical trials database: NCT01827384. https://clinicaltrials.gov

24 Le Tourneau C, Delord JP, Goncalves A et al. Molecularly targeted therapy based on tumour molecular profiling versus conventional therapy for advanced cancer (SHIVA): a multicentre, open-label, proof-of-concept, randomised, controlled Phase 2 trial. Lancet Oncol. 16(13), 1324-1334 (2015).

25 Catenacci DV. Expansion platform type II: testing a treatment strategy. Lancet Oncol. 16(13), 1276-1278 (2015). 